\title{
MULTIOBJECTIVE OPTIMIZATION FOR RISK- BASED MAINTENANCE AND LIFE-CYCLE COST OF CIVIL INFRASTRUCTURE SYSTEMS
}

\author{
D. M. Frangopol, ${ }^{1}$ and M. Liu ${ }^{2}$ \\ ${ }^{1}$ Department of Civil, Environmental, and Architectural Engineering, University of Colorado, \\ Boulder, CO 80309-0428,U.S.A. dan.frangopol@colorado.edu, ${ }^{2}$ Formerly, Department of De- \\ partment of Civil, Environmental, and Architectural Engineering, University of Colorado, Boul- \\ der,CO 80309-0428,U.S.A.minliu@illinoisalumni.org*
}

\begin{abstract}
Reliability and durability of civil infrastructure systems such as highway bridges play a very important role in sustainable economic growth and social development of any country. The bridge infrastructure has been undergoing severe safety and condition deterioration due to gradual aging, aggressive environmental stressors, and increasing traffic loads. Maintenance needs for deteriorating highway bridges, however, have far outpaced available scarce funds highway agencies can provide. Bridge management systems (BMSs) are thus critical to cost-effectively allocate limited maintenance resources to bridges for achieving satisfactory lifetime safety and performance. In existing BMSs, however, visual inspections are the most widely adopted practice to quantify and assess bridge conditions, which are unable to faithfully reflect structural capacity deterioration. Failure to detect structural deficiency due to, for example, corrosion and fatigue, and inability to accurately assess real bridge health states may lead to unreliable bridge management decisions and even enormous safety and economic consequences. In this paper, recent advances in risk-based life-cycle maintenance management of deteriorating civil infrastructure systems with emphasis on highway bridges are reviewed. Methods of predicting lifetime safety and performance of highway bridges with and without maintenance are discussed. Treatment of various uncertainties associated with the complex deterioration processes due to time-dependent loading, environmental stressors, structural resistances, and maintenance actions are emphasized. The bridge maintenance management is formulated as a nonlinear, discrete, combinatorial optimization problem with simultaneous consideration of multiple and conflicting objectives, which address bridge safety and performance as well as long-term economic consequences. The effectiveness of genetic algorithms as a numerical multiobjective optimizer for
\end{abstract}

* The authors gratefully acknowledge the partial financial support of the U.K. Highways Agency, the U.S. National Science Foundation through grants CMS-9912525 and CMS-0217290, and the Colorado Department of Transportation. The opinions and conclusions presented in this paper are those of the writers and do not necessarily reflect the views of the sponsoring organizations.

Please use the following format when citing this chapter:

Author(s) [insert Last name, First-name initial(s)], 2006, in IFIP International Federation for Information Processing, Volume 199, System Modeling and Optimization, eds. Ceragioli F., Dontchev A, Furuta H., Marti K., Pandolfi L., (Boston: Springer), pp. [insert page numbers]. 
producing Pareto-optimal tradeoff solutions is demonstrated. The proposed probabilistic multiobjective optimization BMS is applied at project-level for similar bridges and at network-level for a group of different bridges that form a highway network.

Keywords: System reliability, optimization, civil infrastructure, bridges, genetic algorithms.

\section{Introduction}

Future sustained economic growth and social development of any country is intimately linked to the reliability and durability of its civil infrastructure systems such as highway bridges, which are the most critical but vulnerable elements in highway transportation systems. Highway bridges have been and are constantly subject to aggressive environments and ever-increasing traffic volumes and heavier truckloads, which degrade at an alarming rate the longterm bridge performance. In the United States, nearly $30 \%$ of the 600,000 existing bridges nationwide are structurally deficient or functionally obsolete; the associated costs of maintenance, repair, and replacement are enormous [14].

Deteriorating civil infrastructure leads to increased direct and indirect costs for business and users. Catastrophic failure of civil infrastructures due to natural hazards (e.g. earthquakes, hurricanes, and floods) and manmade disasters (e.g. vehicular collision and explosive blasts due to terrorists' attacks) [15] can cause widespread social and economic consequences. Therefore, timely and adequate maintenance interventions become indispensable to enhance resilience of civil infrastructure to adverse circumstances. This can substantially increase a country's economic competitiveness. In addition to development of advanced inspection and maintenance technologies, methodologies for costeffective allocation of limited budgets to maintenance management of aging and deteriorating civil infrastructure over the life-cycle are urgently needed in order to optimally balance the lifetime performance and life-cycle cost while ensuring structure safety above acceptable levels.

A variety of practical bridge management systems (BMSs) have been developed and implemented in the United States for achieving desirable management solutions to maintain satisfactory bridge infrastructure performance, including BRIDGIT [20] and Pontis [30]. Most existing BMSs, however, utilize the least long-term economic cost criterion [28]. Recently, practicing bridge managers showed that this approach may not necessarily result in satisfactory long-term bridge performance [29]. Additionally, visual inspection is the most widely used practice to determine the condition and performance deterioration of bridges [1]. This highly subjective evaluation technique leads to significant variability in condition assessment [27]. More importantly, the actual level of structure safety against sudden failure and progressive degradation risks cannot be faithfully or accurately described by visual inspection-based bridge condition 
assessment [13]. Accordingly, maintenance decisions made solely on visual inspection results are not necessarily cost-effective and may cause tremendous safety and economic consequences if inadequate or unnecessary maintenance interventions are performed.

In order to resolve the above problems, all necessary long-term performance and expense considerations need to be incorporated into the maintenance management decision-making process. These multiple aspects include bridge performance such as visual inspection-based condition states, computation-based safety and reliability indices, and life-cycle costs such as agency cost and user cost. Unlike the traditional cost minimization approach, the above multiple criteria should be treated simultaneously so that a multiobjective optimization formulation is generated. As a result, the proposed risk/reliability-based maintenance management methodology leads to a group of optimized management solution options, exhibiting tradeoff between reducing life-cycle cost and improving structure performance. This significantly enables bridge managers to actively and preferably compromise structure safety/reliability and other conflicting objectives under budget and/or performance constraints.

In order to make rational decisions in preservation of deteriorating civil infrastructure, it is imperative that sources of uncertainty associated with the deterioration process with and without maintenance be addressed appropriately. These include imperfect description of mechanical loadings and environmental stressors as well as inexact prediction of deteriorating structure performance. There are two general types of uncertainty: aleatory and epistemic. The aleatory uncertainty is caused by inherent variation of structure deterioration due to combined effects of complex traffic loadings and environmental stressors as well as physical aging. The epistemic uncertainty stems from the randomness caused by subjective assumption in evaluating demand and load-carrying capacity of bridges or insufficient knowledge in understanding, for example, deterioration mechanisms. This type of uncertainty may be reduced provided more information is available [12]. Probable maintenance actions over the life cycle add further uncertainty to accurate prediction of time-varying structure performance.

In this paper, recent advances in application of multiobjective optimization techniques to risk-based maintenance management of civil infrastructure, in particular, highway bridges are reviewed. The multiple and competing objective functions of interest include condition, safety and life-cycle cost. Uncertainties associated with the deterioration process with and without maintenance interventions are treated by Monte Carlo simulation and/or structural reliability theory. The basic theory and effectiveness of evolutionary computation techniques such as genetic algorithms (GAs) in solving multiobjective optimization problems are discussed. Two application examples of GA-based bridge maintenance management are provided. The first example deals with project-level 
maintenance management of preserving a large population of similar deteriorating highway reinforced concrete crossheads. The second example is concerned with network-level bridge maintenance management for a number of different bridges that form a highway transportation network.

\section{Multiobjective Optimization Algorithms}

Because bridge management involves scheduling of different maintenance strategies to different bridges at discrete years, it can be readily formulated as a combinatorial optimization problem for which multiple and usually conflicting objectives need to be considered. In this section, the basic concept of multiobjective optimization is presented, the techniques of genetic algorithms (GAs) are discussed, and the application of GAs to the civil infrastructure management problems is emphasized.

\subsection{General Formulation}

A generic multiobjective optimization problem can be stated as

$$
\begin{gathered}
\text { Optimize } \mathbf{f}(\mathbf{x}) \equiv\left[f_{1}(\mathbf{x}), f_{2}(\mathbf{x}), \cdots, f_{m}(\mathbf{x})\right] \\
\text { Subject to } \mathbf{C}(\mathbf{x}) \equiv\left[C_{1}(\mathbf{x}), C_{2}(\mathbf{x}), \cdots, C_{n}(\mathbf{x})\right]<0
\end{gathered}
$$

where $\mathbf{f}$ is a set of objective functions that are usually conflicting in nature; $\mathbf{C}$ is a set of constraints that define the valid solution space; $\mathbf{x}=$ a vector of design variables. Unlike optimization problems with single objectives, there are no unique solutions that can optimize all objectives simultaneously for a multiobjective optimization problem. Instead, a group of Pareto-optimal or nondominated solutions are present, which exhibit the optimized tradeoff in compromising these objectives. A solution $\mathbf{x}^{*}$ is Pareto-optimal if and only if there does not exist another solution that is no worse in all objectives and is strictly better in at least one objective. If all objectives are to be minimized, this can be stated mathematically as

$$
\begin{gathered}
f_{i}(\mathbf{x}) \leq f_{i}\left(\mathbf{x}^{*}\right), \text { for } \mathrm{i}=1,2, \ldots, \mathrm{m} ; \text { and } \\
f_{k}(\mathbf{x}) \leq f_{k}\left(\mathbf{x}^{*}\right), \text { for at least } k \text { th objective. }
\end{gathered}
$$

\subsection{Genetic Algorithms}

Most traditional optimization algorithms are problem-dependent and singleobjective oriented. Gradients are usually utilized to guide the search process and continuous design variables are often assumed. These pose significant difficulties to practical maintenance management problems. In contrast, heuristic algorithms based on evolutionary strategies such as GAs [19], simulated annealing [25], and tabu search [18] are very suitable for practical maintenance scheduling problems. In particular, GAs are stochastic search and optimiza- 
tion engines that follow the survival-of-the-fitness theory from the biological sciences. Since their inception in the 1960's, GAs have been successfully used in a wide array of applications due to their ease of implementation and robust performance for difficult engineering and science problems of vastly different natures. GAs are general-purpose numerical tools and gradients are no longer needed and discrete-valued design variables can be handled without difficulty. More importantly, GAs can handle multiple objectives simultaneously.

GAs usually operate on solutions that are encoded as genotypic representations (i.e. chromosomes) from their original phenotypic representations (i.e. actual data values). GAs start with a set of initial solutions (population) that is randomly generated in the search space. For each solution in the current population, objective functions defining the optimization problem are evaluated and a fitness value is assigned to reflect its (relative) merit standing in the population. Based on the fitness values, GAs perform a selection operation that reproduces a set of solutions with higher fitness values from the previous generation to fill a mating pool. A crossover operation is then pursued with which two parent solutions in the mating pool are randomly selected and interchange, with a prescribed probability, their respective string components at randomly selected bit locations referred to as cross sites. The resulting new solutions are called children or offspring. This step is meant to hopefully combine better attributes from the parent solutions so that child solutions with improved merits could be created. The next operation in GA is mutation that changes the genotype value at one or more randomly selected bit locations in a child solution with another prescribed probability. This operation serves to possibly recover useful information that could by no means be accessible through selection or crossover operation and therefore encourages search into a completely new solution space. After these three basic operations, a new generation is created. The search process continues until prescribed stopping criteria are met.

A successful multiobjective GA must have the ability to obtain a nondominated set of solutions close to the global Pareto-optimal front, and to have this solution set as diverse as possible, that is, to prevent solution clustering from occurring. Note that the selection operation is based on the relative fitness measures of solutions. Unlike single-objective problems where the objective function itself may be used as the fitness measure, after scaling and constrainthandling treatment, a multiobjective GA needs a single fitness measure that reflects the overall merit of multiple objectives. Multiobjective GAs have been fruitfully studied and developed in the last decade [7], many of which adopt Goldberg's nondominated sorting technique [19] to rank all solutions in a population, as discussed in the following.

For a given population of solutions, a nondominated subset is first identified according to the definition of Pareto optimality as defined previously. All solutions in this nondominated subset are assigned a rank of one and are then 
Figure 1. Population ranking based on nondominated sorting



FIRST OBJECTIVE

temporarily deleted from the population. The nondominated subset of the remaining solutions is identified and assigned a rank of two. This procedure continues until all solutions in the population have been assigned appropriate ranks. A solution in the front of a lower-numbered rank is assigned a higher fitness than that of a solution in the front of a higher-numbered rank. As a result, solutions closer to the global Pareto-optimal front have higher fitness values. As an illustration, consider a generic problem that has two objectives to be minimized. Fig. 1 indicates, in the solution space, ten solutions that are classified into four fronts with varied ranks. To assign fitness values to solutions with the same rank, niching strategies are used to determine relative fitness values by, for example, a crowding distance measure [10]. This measure is taken as an average distance of the two solution points on either side of the current solution along each of the objectives and thus serves as an estimate of the density of solutions surrounding a particular solution in the population.

Constraints in the GA-based optimization must be handled appropriately in GAs [6]. One possible approach is to assign to constraint-violating solutions dummy fitness values, which are defined in terms of degrees of constraint violation and are always less than those of valid solutions in the population. Thus the original constrained optimization problems are equivalently converted into unconstrained problems. Alternatively, constraint-violation may be considered by modifying genetic operators instead of assigning fictitious fitness values to invalid solutions. Deb [10] proposed a constrained binary tournament selection scheme that determines from two randomly picked solutions in the population the better solution based on three rules: (i) if both solutions are feasible, the one with higher fitness wins; (ii) if one solution is feasible and the other is infeasible, the feasible one always wins; (iii) if two solutions are both infeasible, the one with less degree of constraint-violation wins.

In multiobjective GAs, elitists usually refer to the generation-wise nondominated solutions. It is beneficial to retain elitist solutions in the subsequent generations for evolution operations due to their excellent genetic properties. 
The stochastic nature of GAs, however, may disturb this ideal situation especially at early generations when the number of elitists is much smaller than the population size. To solve this problem, the elitist strategy may be adopted by forcibly inserting nondominated solutions (elitists) from the last generation back to its offspring population after basic genetic operations (i.e. selection, cross, and mutation) are performed. An updated set of elitists is then identified based on the population augmented by the elitists from the last generation. Previous studies have shown that the elitist scheme plays a crucial role in improving the optimization results [10].

\subsection{GA-Based Maintenance Management}

Research on use of multiobjective optimization techniques in maintenance management of civil infrastructure has appeared recently in the literature. Multiple and conflicting performance indicators such as condition, safety, durability along with life-cycle cost are simultaneously considered as separate criteria $\{21,24,26,17\}$. Interestingly almost all these research activities are conducted using GAs as numerical optimizers. This is because the practical maintenance management problems can be best posed as combinatorial optimization [18]. Due to their inherent features as previously discussed, GAs are very effective for solving these kinds of problems.

Many GAs work with a fixed population size. As generations evolve, the nondominated solutions fill most solution slots in a population, which may make it very difficult for dominated solutions to enter the population for genetic operations. As a result, the diversity of nondominated solutions in the subsequent generations may not be fully explored due to lack of information from valid yet dominated solutions. In this study, the initial GA population consists of 1,000 randomly generated trial solutions and each of the subsequent generations contains 200 offspring solutions plus the nondominated solutions from the previous (i.e. parent) generation. In addition, the fitness value is determined according to Goldberg's nondominated sorting plus Deb's crowding distance measure; Deb's constrained binary tournament selection scheme is adopted; a uniform crossover is applied with a probability of 50\%; a uniform mutation is performed with a probability of $5 \%$. Although this is a relatively high rate of mutation, by using elitism to preserve the nondominated solutions at each generation, mutation tends not to be very disruptive; sometimes a high level of mutation is used to avoid premature convergence.

\section{Bridge Maintenance Management at Project-Level}

Much effort has been devoted by researchers and practitioners to develop methodologies for long-term maintenance management of deteriorating bridges $[8,12,13,16,17]$ Most previous research can be categorized as project-level 
types because only individual bridges or a group of similar bridges are considered. In this section, the time-dependent bridge performance deterioration with and without maintenance interventions is predicted by a continuous computational model [16]. This model describes the performance profiles without maintenance by a curve characterized by an initial performance level, time to damage initiation, and a deterioration curve governed by appropriate functions and, in the simplest form, a linear function with a constant deterioration rate. Effects of a generic maintenance action include prompt performance improvement, deterioration suppression for a prescribed period of time, deterioration severity reduction, and prescribed duration of maintenance effect. Epistemic uncertainties associated with the deterioration process are considered in terms of respective probabilistic distributions of the controlling parameters of this computational model. Monte Carlo simulation is used to account for these uncertainties by obtaining statistical performance profiles of deteriorating structures.

\subsection{Problem Statement}

The GA-based management procedure is used to prioritize maintenance needs for deteriorating reinforced concrete highway crossheads through simultaneous optimization of both structure performance and life-cycle maintenance cost. The maintenance management problem is thus posed as a combinatorial multiobjective optimization problem in that, for any year over the specified time horizon, at most one maintenance strategy may be carried out. Time-dependent performances of these structures are described using appropriate indicators in terms of condition and safety states.

For reinforced concrete elements under corrosion attack in the United Kingdom, Denton [11] categorized visual inspection-based condition states into four discrete levels, denoted as $0,1,2$, and 3 , that represent no chloride contamination, onset of corrosion, onset of cracking, and loose concrete/significant delamination, respectively. A value larger than 3 indicates an unacceptable condition state. As a subjective measure, however, the condition index may not faithfully reflect the true load-carrying capacity of structural members. According to bridge specifications in the United Kingdom, the safety index is defined as the ratio of available to required live load capacity [9]. It is considered unacceptable structure performance if the value of safety index drops below 0.91 .

The goal is to obtain a set of sequences of maintenance actions applied over the specified time horizon that, in an optimized tradeoff manner, (i) decrease the largest (i.e. worst) lifetime condition index value, (ii) increase the smallest (i.e. worst) lifetime safety index value, and (iii) decrease the present value of lifecycle maintenance cost. The constraints are enforced such that the condition 
index value must be always less than 3.0 and the safety index value must be always greater than 0.91 .

Five maintenance strategies are considered: replacement of expansion joints, silane, cathodic protection, minor concrete repair, and rebuilding [11,22]. Replacement of expansion joints is statistically the least costly. It does not improve performance or delay deterioration but alleviates deterioration severity of both condition and safety performance. The silane treatment reduces chloride penetration but does not correct existing defects or replace deteriorated structural components. Statistically speaking, silane reduces deterioration of condition more efficiently than replacement of expansion joints while having the same effects on safety deterioration. Cathodic protection replaces anodes and thus suppresses corrosion of reinforcing bars almost completely. It postpones deterioration of both condition and safety for 12.5 years upon application. The minor concrete repair strategy is applied to replace all cover concrete with visual defects but not corroded reinforcing bars. The rebuilding strategy improves both condition and safety levels to those values typical of a new structural component.

\subsection{Numerical Results}

In the numerical implementation, Monte Carlo simulation with a sample size of 1,000 is used to consider effects of uncertainty on prediction of both structure performance and life-cycle maintenance cost. All three objective functions are evaluated in terms of sample mean values. The service life is considered 50 years and the monetary discount rate is $6 \%$. A number of different optimized maintenance planning solutions are generated. These solutions represent the optimized tradeoff among the condition, safety, and life-cycle maintenance cost objectives. Three representative maintenance solutions with different levels of performance enhancement and maintenance needs are shown in Fig. 2. Detailed information can be found in [22].

\section{Bridge Maintenance Management at Network-Level}

Compared to the above project-level maintenance management, a transportation network-oriented methodology provides more rational solutions because the ultimate objective of maintenance management is to improve performance of the entire transportation network instead of merely that of individual structures in the network. In this section, performance evaluation of deteriorating bridge networks is discussed and network-level maintenance management is presented and illustrated with numerical examples.

\section{Problem Statement}

The network reliability measures the level of satisfactory network performance. Most studies on assessment of reliability for transportation highway 
Figure 2. Tradeoff of three project-level maintenance-scheduling solutions
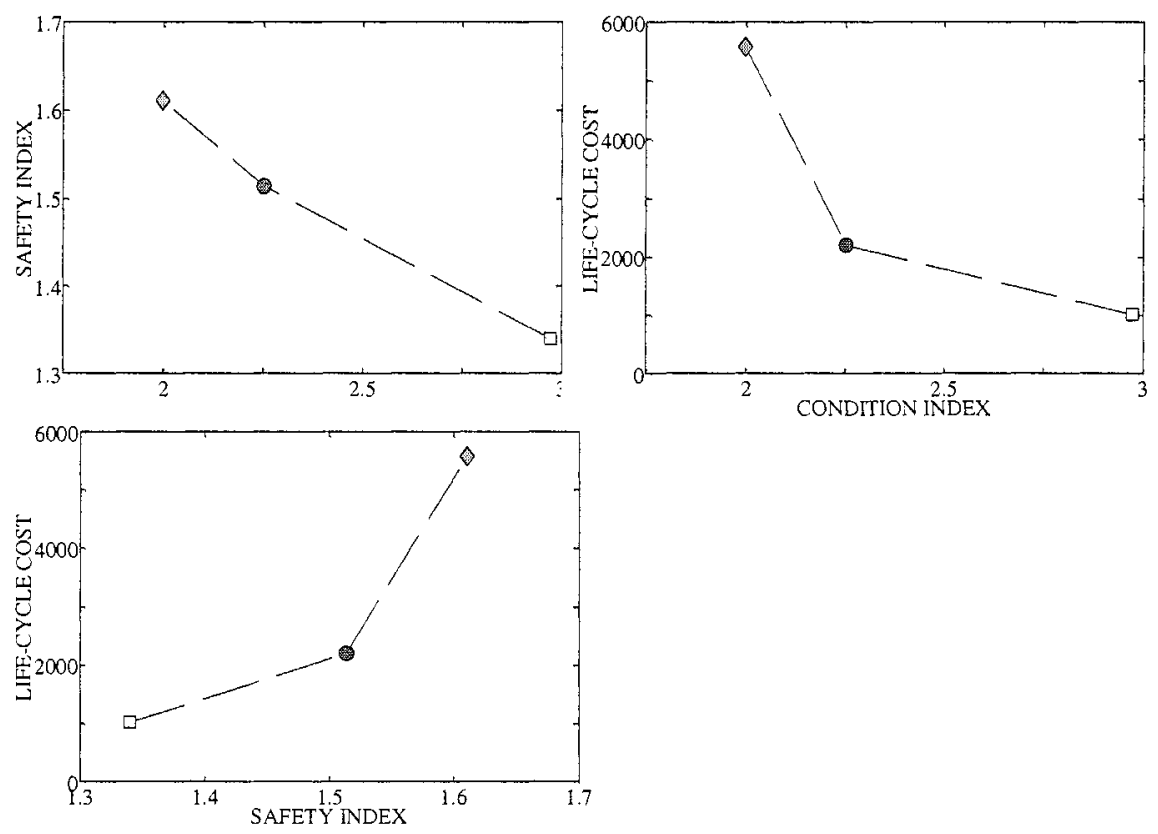

infrastructures have focused on maintenance management of deteriorating road networks for which a travel path consists of multiple links (i.e. roadways between any two nodes) with binary states (either operational or failed). There are three network reliability measures with ascending levels of sophistication: connectivity reliability, travel time reliability, and capacity reliability [4,5]. The connectivity reliability is associated with the probability that nodes in a highway network are connected; in particular, the terminal connectivity refers to the existence of at least one operational path that connects the origin and destination (OD) nodes of interest. The travel time reliability indicates the probability that a successful trip between a specified OD pair can be made within given time interval and level-of-service. Based on this reliability measure, the appropriate level of service that should be maintained in the presence of network deterioration can be determined. The third measure is the capacity reliability, which reflects the possibility of the network to accommodate given traffic demands at a specified service level. In this formulation, link capacities may be treated as random variables to consider the time-dependent probabilistic capacity deterioration. Inherent in the last two reliability measures are the determination of risk-taking route choice models for simulating travelers' behavior in the presence of both perception error and network uncertainty [5]. 
For maintenance management of deteriorating highway networks, it is also very important to use economic terms as a measure of the overall network performance. There are two basic types of costs: agent cost and user cost. The agent cost is composed of direct material and labor expenses needed to perform routine and preventive maintenance, rehabilitation, and replacement of existing transportation facilities. The indirect user costs are caused by loss of adequate service due to, for example, congestion and detour. In some situations the user cost may be a dominating factor in evaluating the overall life-cycle costs for a transportation network. The uncertainty associated with capacity degradation and demand variation should be integrated in the analysis in order to obtain a reliable cost measure.

In this study, the goal of network-level maintenance management is to prioritize maintenance needs to bridges that are of most importance to the network performance and over the specified time horizon. The overall goal is to satisfy the following two requirements in a simultaneous and balanced manner: (i) the overall bridge network performance, which is measured by the lowest level of the lifetime reliability of connectivity between the origin and destination locations, is improved, and (ii) the present value of total life-cycle maintenance cost is reduced.

Four different maintenance strategies are considered herein for enhancing bridge network performance in terms of reliability levels of deteriorating reinforced concrete bridge deck slabs: resin injection, slab thickness increasing, steel plate attaching, and complete replacement [17]. Resin injection is the least costly maintenance type among the four options. It injects epoxy resin into voids and seals cracks in concrete, which repairs the aging deck slabs by reducing the corrosion of reinforcement due to exposure to the open air. The reduction rate in reliability deterioration is assumed 0.03 year for 15 years. The other three maintenance strategies instantly improve the bridge reliability level by various amounts upon application. Increasing slab thickness and attaching steel plate increase the system reliability indices by a maximum of 0.7 and 2.0 , respectively, with unit costs being US $\$ 300 / \mathrm{m}^{2}$ and US $\$ 600 / \mathrm{m}^{2}$, respectively. The complete replacement option restores the structural system to the initial reliability level with a unit cost of US $\$ 900 / \mathrm{m}^{2}$.

\section{$5.1 \quad$ Numerical Results}

The network-level maintenance management is illustrated using a real bridge network in Colorado [3]. This network consists of thirteen bridges of different types. The network performance is evaluated in terms of the terminal reliability for connectivity between two designated locations. Flexure failure of bridge slabs is considered as the only failure mode [2]. Deterioration of reinforcement is caused by deicing chemicals related corrosion. The life cycle is consid- 
Figure 3. Tradeoff of three network-level maintenance-scheduling solutions
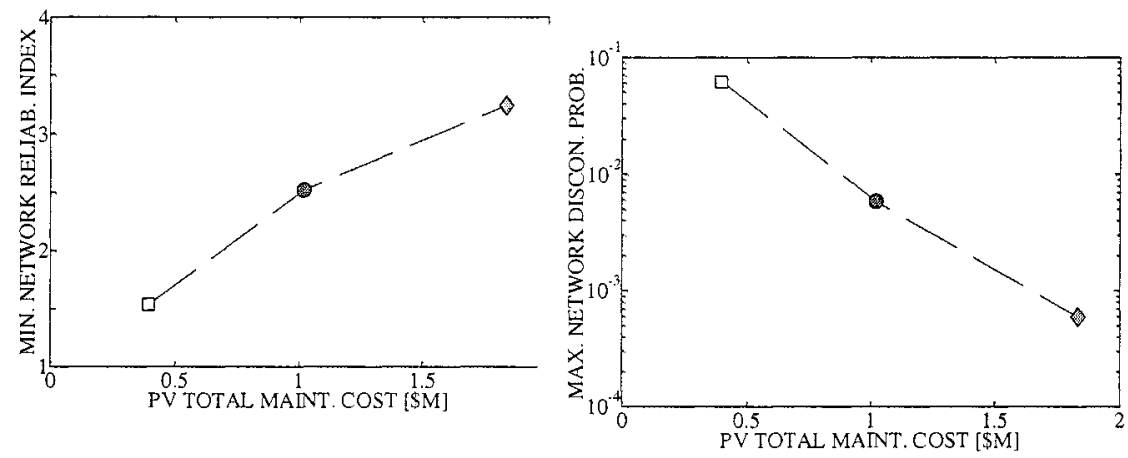

ered 30 years and the discount rate is $6 \%$. The optimized solutions by GA represent a wide spread tradeoff between the conflicting network connectivity (equivalently network disconnectivity probability) and the total maintenance cost objectives. Tradeoff of three representative solutions is plotted in Fig. 3. Detailed information can be found in [23].

\section{Monitoring-Integrated Maintenance Manage- ment}

It is interesting and challenging to integrate the recent developments of structural health monitoring (SHM) technologies into intelligent maintenance management of civil infrastructure systems. Utilizing advanced sensing/information technology and structural modeling/identification schemes, SHM detects, locates, and quantifies structural damages caused by catastrophic natural or manmade events as well as by long-term deterioration. These data assist bridge managers in assessing the health of existing bridges and thus in determining immediate or future maintenance needs for safety consideration and lifespan extension. Most existing research and practice in BMS and SHM, however, are carried out in a disconnected manner. Therefore, a unified framework is necessary to bridge this gap between these two research areas.

Research in these areas represents a crucial step toward improving the traditional approach to BMSs by providing bridge managers with an efficient tool to make timely and intelligent decisions on monitoring, evaluation, and maintenance of deteriorating highway bridges. This can be achieved by exploring the interaction between SHM and BMS strategies in terms of whole-life costing and structural safety/health/reliability. Prediction of time-dependent bridge performance with monitoring is essential in this endeavor. With monitored data, the time-dependent performance will be more reliably estimated and the mainte- 
Figure 4. Bridge performance profiles with and without monitoring-integrated maintenance interventions
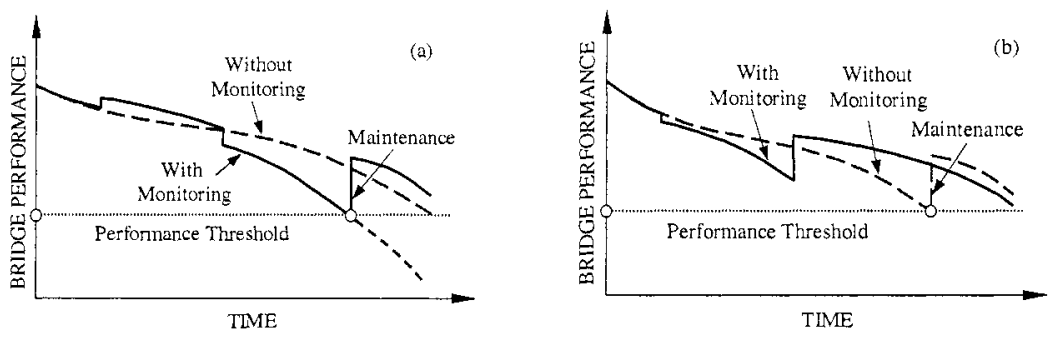

nance interventions will be more accurately applied than in the case without monitoring. Fig. 4 schematically illustrates the influence of monitoring actions on the prediction of bridge performance and on the ensuing maintenance interventions. In Fig. 4(a), with sensed data, earlier, reaching the prescribed performance threshold is predicted, which incurs a timely maintenance intervention. Otherwise, if based on the non-monitoring performance prediction, the maintenance would not have been applied, which would cause tremendous risk concerns and consequences due to failure occurrence. Fig. 4(b) indicates another situation where the monitoring-enriched performance prediction makes unnecessary the maintenance actions predicted by the non-monitoring profile; in this case savings of maintenance costs can be enormous. Therefore, interactions among maintenance, monitoring, and management must be accurately analyzed in order to maintain bridges in timely and economical manners.

\section{Conclusions}

This paper reviews recent developments of risk-based maintenance management of civil infrastructure systems especially of highway bridges, emphasizing simultaneous consideration of multiple criteria related to long-term structure performance and life-cycle cost. Sources of uncertainty associated with the deterioration process are considered in probabilistic performance prediction of structures with and without maintenance interventions. The usefulness of genetic algorithms in solving the posed combinatorial multiobjective optimization problems is discussed. Two illustrative numerical examples are provided. The first example deals with project-level maintenance scheduling for a group of deteriorating reinforced concrete crossheads over a specified time horizon. Structure performance measures, in terms of visually inspected condition and computed load-carrying safety indices, and the present value of long-term maintenance cost are treated as competing objectives. The second example is associated with network-level bridge maintenance management, in which a group of spatially distributed bridges that form a highway network is studied. The 
overall network performance is assessed in terms of the terminal connectivity reliability. A maintenance solution contains a sequence of maintenance interventions that are scheduled at discrete years to be applied to different bridges. The conflicting objectives of the network connectivity reliability and the total maintenance cost are subject to balanced optimization. A set of alternative solutions is produced that exhibits the best possible tradeoff among all competing objectives. Bridge managers' preference on the balance between the lifetime performance and life-cycle cost can be integrated into the decision-making process. Finally, research needs of integrating bridge management systems and structural health monitoring are discussed and illustrated.

\section{References}

[1] AASHTO. Manual for Condition Evaluation of Bridges. 2nd edn. American Association of State Highway and Transportation Officials, Washington, D.C., 1994.

[2] AASHTO. Standard Specifications for Highway Bridges. 16th edn. American Association Of State Highway And Transportation Officials, Washington, D.C., 1996.

[3] F. Akgül, D. M. Frangopol. Rating and Reliability of Existing Bridges in a Network. Journal of Bridge Engineering, ASCE 8(6), 383-393, 2003.

[4] M. G. H. Bell, Y. Iida. Transportation Network Analysis. Wiley, Chichester, 1997.

[5] A. Chen, W. W. Recker. Considering Risk Taking Behavior in Travel Time Reliability, Report No. UCI-ITS-WP-00-24, Institute of Transportation Studies, University of California, Irvine, California, 2000.

[6] C. A. Coello Coello Theoretical and Numerical Constraint-Handling Techniques Used with Evolutionary Algorithms: a Survey of the State of the Art." Computer Methods in Applied Mechanics and Engineering 191, 1245-1287, 2002.

[7] C:A. Coello Coello, D.A. van Veldhuizen, G.B. Lamont. Evolutionary Algorithms for Solving Multi-Objective Problems. Kluwer Academic Publishers, New York, 2002.

[8] P.C. Das. Prioritization of Bridge Maintenance Needs. In: Frangopol, D.M. (ed.): Case Studies in Optimal Design and Maintenance Planning Of Civil Infrastructure Systems. ASCE Reston, 26-44, 1999.

[9] DB12/01. The Assessment of Highway Bridge Structures. Highways Agency Standard for Bridge Assessment, London, 2001.

[10] K. Deb. Multi-Objective Optimization Using Evolutionary Algorithms. Wiley, Chichester, 2001.

[11] S. Denton. Data Estimates for Different Maintenance Options for Reinforced Concrete Cross Heads (Personal communication). Parsons Brinckerhoff Ltd, Bristol, 2002.

[12] Enright, M. P., Frangopol, D. M. Condition Prediction of Deteriorating Concrete Bridges Using Bayesian Updating. Journal of Structural Engineering, ASCE 125(10)(1999) 1118 1125.

[13] A.C. Estes, D.M. Frangopol. Updating Bridge Reliability Based on Bridge Management Systems Visual Inspection Results. Journal of Bridge Engineering, ASCE 8(6), 374-382, 2004.

[14] FHWA. The Status of The Nation's Highways, Bridges, and Transit: Conditions and Performance. U.S. Federal Highway Administration, Washington, D.C., 2002. 
[15] FHWA. Recommendations for Bridge and Tunnel Security. U.S. Federal Highway Administration, Washington, D.C., 2003.

[16] D. M. Frangopol, J S. Kong, E. S.Gharaibeh. Reliability-Based Life-Cycle Management of Highway Bridges. Journal of Computing In Civil Engineering, ASCE 15(1), 27-34, 2001.

[17] H. Furuta, T. Kameda, Y. Fukuda, D.M. Frangopol. Life-Cycle Cost Analysis for Infrastructure Systems: Life Cycle Cost vs. Safety Level vs. Service Life. In: Frangopol, D.M., Brühwiler, E., Faber, M.H., Adey B. (eds.): Life-Cycle Performance of Deteriorating Structures: Assessment, Design and Management. ASCE Reston, Virginia, 19-25, 2004.

[18] F. Glover, M. Laguna. Tabu Search. Kluwer Academic Publishers, New York, 1997.

[19] D. E. Goldberg. Genetic Algorithms in Search, Optimization and Machine Learning. Addison-Wesley, Reading, 1989.

[20] H. Hawk, E. P. Small. The BRIDGIT Bridge Management System. Structural Engineering International, IABSE 8(4), 309-314, 1998.

[21] C. Liu, A. Hammad, Y. Itoh,: Multiobjective Optimization of Bridge Deck Rehabilitation Using a Genetic Algorithm. Computer-Aided Civil and Infrastructure Engineering, 12 (1 431-443, 1997.

[22] M. Liu, D. M. Frangopol. Bridge Annual Maintenance Prioritization under Uncertainty by Multiobjective Combinatorial Optimization. Computer-Aided Civil and Infrastructure Engineering, 20(5), 343-353, 2005.

[23] M. Liu, D. M. Frangopol. Balancing the Connectivity Reliability of Deteriorating Bridge Networks and Long-Term Maintenance Cost Using Optimization. Journal of Bridge Engineering, ASCE 10(4), 468-481, 2005.

[24] Z. Lounis, D. J. Vanier. A Multiobjective and Stochastic System for Building Maintenance Management. Computer-Aided Civil and Infrastructure Engineering. 15 (2000) 320-329.

[25] R. Marett, M. Wright. A Comparison of Neighborhood Search Techniques for Multiobjective Combinatorial Problems. Computers \& Operations Research. 23(5), 465-483, 1996.

[26] A. Miyamoto, K. Kawamura, H. Nakamura. Bridge Management System and Maintenance Optimization for Existing Bridges. Computer-Aided Civil and Infrastructure Engineering. $15,45-55,2000$.

[27] M. E. Moore, B. M. Phares, B. A. Graybeal, D. D. Rolander, G. A. Washer. Reliability of Visual Inspection for Highway Bridges (Report Nos. FHWA-RD-01-020 and FHWARD-01-21). U.S. Federal Highway Administration, Washington, D.C., 2001.

[28] NCHRP. Bridge Life-Cycle Cost Analysis (Report 483). National Cooperative Highway Research Program, Transportation Research Board, Washington, D.C., 2003.

[29] R. W. Shepard, M. B. Johnson, W. E. Robert, A .R. Marshall. Modeling Bridge Network Performance - Enhancing Minimal Cost Policies. In: Watanabe, E., Frangopol, D.M., Utsunomiya, T. (eds.): Bridge Maintenance, Safety, Management and Cost. A. A. Balkema Publishers, Leiden,Book and CD-ROM, 2004.

[30] P.D. Thompson, E.P. Small, M. Johnson, A.R. Marshall. The Pontis Bridge Management System. Structural Engineering International, IABSE. 8(4),303-308, 1998. 